\title{
Additional Value of Diffusion-Weighted Imaging to Evaluate Prognostic Factors of Breast Cancer: Correlation with the Apparent Diffusion Coefficient
}

\author{
Eun Kyung Park, Kyu Ran Cho, ${ }^{1, *}$ Bo Kyoung Seo, ${ }^{2}$ Ok Hee Woo, ${ }^{3}$ Sung Bum Cho, ${ }^{1}$ and Jeoung \\ Won $\mathrm{Bae}^{4}$ \\ ${ }_{1}^{1}$ Department of Radiology, Anam Hospital, Korea University College of Medicine, Seoul, Korea \\ Department of Radiology, Anam Hospital, Korea University College of Medicine, Seoul, Korea
2Department of Radiology, Ansan Hospital, Korea University College of Medicine, Gyeonggi-do, Korea \\ ${ }_{3}^{3}$ Department of Radiology, Ansan Hospital, Korea University College of Medicine, Gyeonggi-d \\ ${ }^{4}$ Department of Radiology, Guro Hospital, Korea University College of Medicine, Seoul, Korea
${ }^{2}$ Department of Surgery, Anam Hospital, Korea University College of Medicine, Seoul, Korea \\ ${ }^{*}$ Corresponding author: Kyu Ran Cho, Department of Radiology, Korea University College of Medicine 73, Inchon-ro, Seongbuk-gu, Seoul 02841, Korea. Tel: +82-29205578, Fax: \\ +82-29293796, E-mail: krcho@korea.ac.kr \\ Received 2015 September 15; Revised 2015 October 21; Accepted 2015 October 26.
}

\begin{abstract}
Background: Breast cancer is a heterogeneous disease with diverse prognoses. The main prognostic determinants are lymph node status, tumor size, histological grade, and biological factors, such as hormone receptors, human epidermal growth factor receptor 2 (HER2), Ki-67 protein levels, and p53 expression. Diffusion-weighted imaging(DWI) can be used to measure the apparent diffusion coefficient(ADC) that provides information related to tumor cellularity and the integrity of the cell membranes.

Objectives: The goal of this study was to evaluate whether ADC measurements could provide information on the prognostic factors of breast cancer.

Patients and Methods: A total of 71 women with invasive breast cancer, treated consecutively, who underwent preoperative breast MRIs with DWI at 3.0 Tesla and subsequent surgery, were prospectively included in this study. Each DWI was acquired with $b$ values of 0 and $1000 \mathrm{~s} / \mathrm{mm}^{2}$. The mean ADC values of the lesions were measured, including the entire lesion on the three largest sections. We performed histopathological analyses for the tumor size, lymph node status, histological grade, hormone receptors, human epidermal growth factor receptor 2 (HER2), Ki-67, p53, and molecular subtypes. The associations with the ADC values and prognostic factors of breast cancer were evaluated using the independent-samples $t$ test and the one-way analysis of variance (ANOVA).

Results: A low ADC value was associated with lymph node metastasis $(\mathrm{P}<0.01)$ and with high Ki-67 protein levels $(\mathrm{P}=0.03)$. There were no significant differences in the ADC values among the histological grade $(\mathrm{P}=0.48)$, molecular subtype $(\mathrm{P}=0.51)$, tumor size $(\mathrm{P}=0.46)$, and p53 protein level $(\mathrm{P}=0.62)$.

Conclusion: The pre-operative use of the 3.0 Tesla DWI could provide information about the lymph node status and tumor proliferation for breast cancer patients, and could help determine the optimal treatment plan.
\end{abstract}

Keywords: Breast Cancer, Diffusion-Weighted Imaging, Apparent Diffusion Coefficient, Breast Magnetic Resonance

\section{Background}

Breast cancer is a heterogeneous disease with distinct molecular subtypes (luminal A, luminal B, HER2 positive, and triple-negative), which have diverse clinical outcomes and treatment responses. The luminal B subtype shows a higher proliferation of tumor cells and poorer prognosis than the luminal A subtype (1). The HER2 positive and triple-negative breast cancers are associated with a worse prognosis when compared to the luminal subtypes (2). Moreover, the lymph node status, tumor size, and histological grade are the three main prognostic determinants in breast cancer. Based on these clinical and histological factors, the biological factors, such as the hormone receptors and human epidermal growth factor receptor 2(HER2), Ki-67 protein levels, and p53 expression are strongly associated with the prognosis and outcomes in breast cancer (3). These are major considerations in the management of patients with breast cancer.

The diagnosis and preoperative staging of breast cancer are made on the basis of mammography, ultrasonography, and dynamic contrast-enhanced magnetic resonance imaging (DCE-MRI). Diffusion-weighted imaging (DWI) has become a helpful tool in providing additional information for the characterization of the lesions detected by breast MRIs (4-6). DWI can be used to measure the apparent diffusion coefficient (ADC), a quantitative measure of the diffusivity of water, which provides information related to tumor cellularity and the integrity of the cell membranes (7). Several studies have evaluated the associations of the ADC value with those of the lymph node status, tumor size, histological grade, and

Copyright (C) 2016, Tehran University of Medical Sciences and Iranian Society of Radiology. This is an open-access article distributed under the terms of the Creative Commons Attribution-NonCommercial 4.0 International License (http://creativecommons.org/licenses/by-nc/4.0/) which permits copy and redistribute the material just in noncommercial usages, provided the original work is properly cited. 
biological factors, but their results were inconclusive (812). Recently, a few studies reported their results for the $\mathrm{ADC}$ value and prognostic factors of breast cancer, using an ultra-high-field (3.0 Tesla) MRI $(9,13)$; however, to our knowledge, there has been no prospective study of the diagnostic value of the ADC measurements from a 3.0 Tesla MRI in determining the prognosis of breast cancer.

\section{Objectives}

The purpose of this study was to evaluate the ADC values of invasive breast cancer, and to investigate whether the use of DWI for these measures could provide information about the prognostic factors in breast cancer, including the lymph node status, tumor size, histological grade, hormone receptors, and HER2, Ki-67, and p53 protein expressions.

\section{Patients and Methods}

\subsection{Patients}

This prospective study was approved by the institutional review board, and informed consent was waived. A total of 112 women with biopsy-proven breast cancer scheduled to undergo surgery were enrolled in our study, between July 1, 2013 and April 30, 2014. The patients were examined using DWIs with b values of 0 and $1000 \mathrm{~s} / \mathrm{mm}^{2}$ added to the conventional DCE-MRI. Only the largest diameter lesion per patient was evaluated.

Patients that met any of the following criteria were excluded from this study: pure ductal carcinoma in situ (n $=10)$, lesion size $<5 \mathrm{~mm}(\mathrm{n}=1)$, motion artifact in DWI $(n=5)$, undergoing neoadjuvant chemotherapy $(n=16)$, excisional biopsy prior to MRI $(\mathrm{n}=5)$, and not undergoing surgery $(n=3)$. One patient was excluded because the percutaneous biopsy determined that the lesion was an invasive ductal carcinoma, but surgery confirmed it to be an adenomyoepithelioma.

\subsection{MR Image Acquisition}

A breast MRI was performed by using a 3.0 Tesla MRI system (Achieva 3.0T TX; Philips Healthcare, Andover, MA), with a dedicated phased-array breast coil, and the subjects in the prone position. The DWI was acquired in the transverse plane, covered both breasts, and a spinecho single-shot echo planar imaging sequence with a diffusion-sensitizing gradient was applied orthogonally. These images were used to synthesize the isotopic transverse images (repetition time (ms)/echo time (ms): 5417/72; b values: $0,1000 \mathrm{~s} / \mathrm{mm}^{2}$; image matrix: $96 \times 126$; field of view: $320 \times 320 \mathrm{~mm}$; section thickness: $3 \mathrm{~mm}$; section gap: $0 \mathrm{~mm}$; 3-signal acquired; acquisition time: $80 \mathrm{~s})$. We chose the b value of $1000 \mathrm{~s} / \mathrm{mm}^{2}$ on the basis of the recommendations of the expert groups. After the DWI, T2-weighted fat spin-echo transverse images were also obtained, using the following image parameters: repetition time (ms)/echo time (ms): 5727/70; flip angle: $90^{\circ}$; image matrix: $620 \times 309$; field of view: $581 \times 342 \mathrm{~mm}$; section thickness: $3 \mathrm{~mm}$; and section gap: $0 \mathrm{~mm}$.

A 3-dimensional T1-weighted fast-spoiled gradient-echo sequence was also performed, with the transverse imaging of one pre-contrast and six post-contrast dynamic series, using Gadoterate meglumine (Dotarem; Guerbet, Villepinte, France) as a contrast agent, immediately after and at $60,120,180,240$, and 300 s after the contrast injection. The image parameters were: repetition time (ms)/ echo time (ms): 6/3; flip angle: $0^{\circ}$; image matrix: $436 \times$ 436; field of view: $330 \times 340 \mathrm{~mm}$; section thickness: $3 \mathrm{~mm}$; and section gap: $1.5 \mathrm{~mm}$. Gadoterate meglumine was injected at $0.1 \mathrm{~mL}$ per kilogram of body weight, from an antecubital vein using a power injector (Spectris; Medrad, Indianola, PA), at a rate of $2 \mathrm{~mL} / \mathrm{s}$. For the DCE-MRI, we performed post-processing, including early subtraction by subtracting the pre-contrast images from the first postcontrast images, calculation of time-intensity curves of enhancing regions, and generation of maximum intensity projection images.

\subsection{DWI and ADC Analysis}

The ADC maps were constructed using software provided by the MRI system manufacturer (Phillips Medical Systems, Andover, MA) and the following equation:

$$
\mathrm{ADC}=\sum_{i=1}^{n} \frac{\ln \left(\frac{s_{i}}{s_{0}}\right)}{b_{i}}
$$

Where, $\mathrm{b}_{\mathrm{i}}$ is the diffusion gradient value and

$$
b=\gamma^{2} G^{2} \delta^{2}\left(\Delta-\frac{\delta}{3}\right)
$$

Where, $\gamma$ is the gyromagnetic ratio; $G$, the gradient strength; $\delta$, the diffusion gradient duration; and $\Delta$, the time between the diffusion gradient pulses $\left(S_{0}\right.$ is the first acquired image [with $b=0$ ], and $S_{i}$ is the ith image). After a hypointense lesion was identified on an ADC map, the regions of interest (ROIs) were drawn manually to cover the entire tumor in the three largest cross-sections, by using the DCE-MRI information for reference purposes, via CAD stream imaging software version 5.2 (Merge Healthcare, Chicago, IL). If the lesion was small, to identify it in the three different cross-sectional planes, ROIs were drawn repetitively in the largest plane. We took care to avoid the regions of high T2 within a lesion, such as cyst, hematoma, necrosis, normal breast parenchyma, or fat. The mean ADC of each ROI was determined, and an average ADC was calculated for each lesion (Figure 1).

To evaluate the intraobserver variability, the radiologist re-measured the ADC values in 30 cases in random order, 9 weeks after the first measurements, and the Kappa statistics were evaluated. 

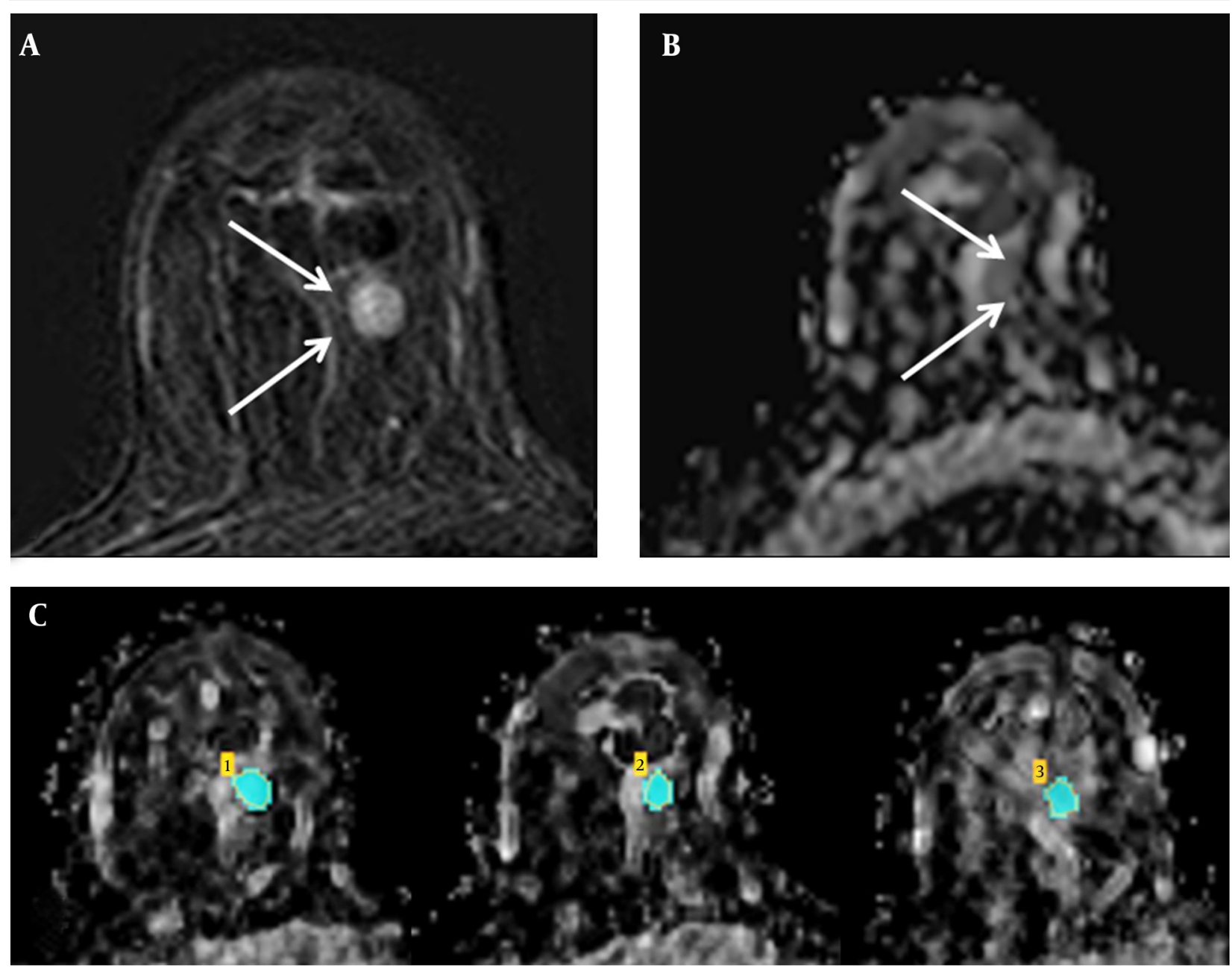

Figure 1. Measuring the ADC value in breast cancer using the CAD system. A, Axial contrast-enhanced T1-weighted subtraction MR image showing enhanced mass in the right breast (arrows); B, Axial ADC map (b values: 0 and $1000 \mathrm{~s} / \mathrm{mm}^{2}$ ) showing the same lesion (arrows) with restricted diffusion; C, On the axial ADC map, three regions of interest were manually drawn to include the entire tumor in the three largest cross-sectional planes, and the mean ADC value was calculated.

\subsection{Histopathological Analysis}

The histological grades and details of the estrogen receptors (ER), progesterone receptors (PR), and HER2 expressional statuses were obtained from post-operative histopathological reports. The histopathological findings were reviewed by two pathologists specializing in mammary tissue, in consensus. The histological grades were assessed using the numerical scoring system for the tubule formation, pleomorphism, and mitotic counts described by Elston and Ellis (14). The total score could range from 3 to 9 , with a scores 3 to 5 representing grade 1 , scores 6 and 7 were grade 2, and 8 and 9 were grade 3 . Following Black et al. (15), the statuses of the ER and PR were considered using the Allred scoring system, and the results of the HER2 expression were recorded as $0,1+, 2+$, or $3+$, according to the manufacturer's recommendations. Tumors with HER2 expressions of 0 or $1+$ were classified as negative, and $3+$ was positive. The tumors with an HER2 expression of 2+ were studied by fluorescent in situ hybridization to determine the HER2 gene amplification.

According to the receptor status, the immunohistochemical tumor subtype was classified into luminal A, luminal B, HER2 positive, and triple negative breast cancer. The Ki-67 protein expression was evaluated by using the percentage of immunoreactive cells from 1000 tumor cells in the hot spots, and the mean percentage was used as a representative value. The Ki-67 index was determined to be positive if the expression was $\geq 14 \%$, and p53 was considered to be positive if the expression was $\geq 10 \%$. The presence of axillary lymph node metastasis was also recorded. The lymph node specimens were obtained by sentinel lymph node dissection, and if the sentinel lymph node was positive, immediate axillary lymph node dissection was also performed. The presence of metastasis, including micrometastasis, was regarded as a positive 
finding, while the presence of isolated tumor cells in the lymph nodes was regarded as a negative finding.

\subsection{Statistical Analysis}

To determine whether the ADC values followed a normal distribution, the Kolmogorov-Smirnov test was performed. The ADC values were compared according to the histological type; lymph node status; tumor size; ER, PR, and HER2 statuses; Ki67 index; and p53 expression, using the independent-samples t test. For the statistical analysis, the tumor sizes were measured by DCE subtraction MRI and classified into two categories ( $\geq 2 \mathrm{~cm}$ or $<2 \mathrm{~cm}$ ). We also compared the ADC values according to the histological grade and immunohistochemical type, using the one-way analysis of variance (ANOVA). Statistical software (SPSS, IBM Corp., Released 2011, IBM SPSS Statistics for Windows, Version 20.0. Armonk, NY) was used for all data analyses, and a P value of less than 0.05 was considered to be a significant difference.

\section{Results}

\subsection{Clinical Data}

Seventy-one women met our inclusion and exclusion criteria, and the mean \pm SD age of these patients was $54 \pm 11$ years (age range: 32 - 74 years). Of the 71 patients, 54 (76\%) were diagnosed with invasive ductal carcinoma not otherwise specified, and 17 (24\%) had special types of invasive ductal carcinomas (invasive lobular carcinoma, 4; invasive micropapillary carcinoma, 3 ; mucinous carcinoma, 2; medullary carcinoma, 2; cribriform carcinoma, 2; neuroendocrine carcinoma, 2; metaplastic carcinoma, 1 ; and tubular carcinoma, 1). The lesion sizes ranged from 0.6 to $10.1 \mathrm{~cm}$ (mean \pm SD: $2.0 \pm 1.4 \mathrm{~cm}$ ). For 24 (34\%) of 71 tumors, the patients underwent mastectomy, and 47 (66\%) were treated with breast-conserving therapy.

\subsection{ADC Values and Prognostic Factors}

According the Kappa statistics, perfect intraobserver agreement was demonstrated $(\mathrm{P}=0.97)$. The mean ADC value of the 71 tumors was $0.91 \pm 0.20 \times 10^{-3} \mathrm{~mm}^{2} / \mathrm{s}$ (range: $\left.0.58-1.44 \times 10^{-3} \mathrm{~mm}^{2} / \mathrm{s}\right)$. The mean ADC values of those invasive ductal carcinomas not otherwise specified, and the special types of invasive ductal carcinoma were not significantly different $\left(0.89 \pm 0.16 \times 10^{-3} \mathrm{~mm}^{2} / \mathrm{s}\right.$ versus $0.98 \pm$ $\left.0.23 \times 10^{-3} \mathrm{~mm}^{2} / \mathrm{s}, \mathrm{P}=0.08\right)$. The mean ADC value was not statistically different between histologic grade and tumor size subgroups ( $\mathrm{Ps}=0.19$ and 0.46 respectively). Moreover, there was no significant differences among the mean ADC values of different subgroups determined by ER, PR and HER2 status (Ps $=0.93,0.88$ and 0.85 respectively).

The mean ADC of the invasive breast cancers with lymph node metastasis was $0.83 \pm 0.13 \times 10^{-3} \mathrm{~mm}^{2} / \mathrm{s}$, and that of the invasive breast cancers without lymph node metastasis was $0.94 \pm 0.21 \times 10^{-3} \mathrm{~mm}^{2} / \mathrm{s}$. The mean ADC of the invasive carcinomas with lymph node metastasis was significantly lower than that of the invasive carcinomas without lymph node metastasis $(\mathrm{P}<0.01)$ (Figures 2 and 3 ). The mean ADC of the high Ki-67 cancers was significantly lower than that of the Ki67-negative cancers $\left(0.85 \pm 0.15 \times 10^{-3} \mathrm{~mm}^{2} / \mathrm{s}\right.$ versus $0.96 \pm 0.22 \times 10^{-3} \mathrm{~mm}^{2} / \mathrm{s}, \mathrm{P}=0.03$ ) (Figure 4 and 5).

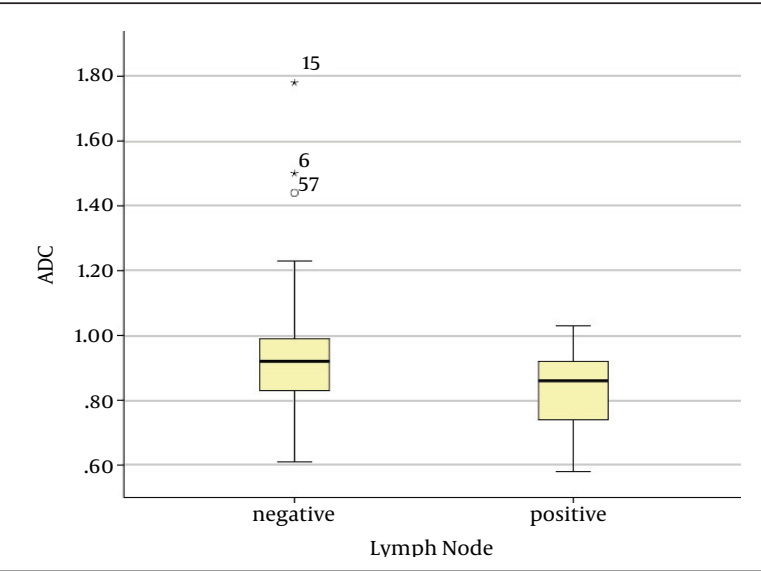

Figure 2. Graph showing the mean $A D C$ values of invasive breast cancers according to lymph node status. The mean ADC value of the invasive breast cancer with lymph node metastasis $\left(0.83 \pm 0.13 \times 10^{-3} \mathrm{~mm}^{2} / \mathrm{s}\right)$ was significantly lower than that without lymph node metastasis $(0.94 \pm 0.21$ $\times 10^{-3} \mathrm{~mm}^{2} / \mathrm{s} ; \mathrm{P}<0.01$ ). The two extreme values in the graph (asterisks) were the ADC values of the mucinous carcinomas with no axillary lymph node metastasis.


Figure 3. Findings in the left breast of a 74-year-old woman with a diagnosis of left breast cancer. A, Axial contrast-enhanced T1-weighted subtraction MR image of the left breast showing irregular enhancing mass (arrows), $10 \times 9 \mathrm{~mm}$ in size; B, Diffusion-weighted image (b value: $1000 \mathrm{~s} / \mathrm{mm}^{2}$ ); $\mathrm{C}$, Axial ADC map (b values: 0 and $1000 \mathrm{~s} / \mathrm{mm}^{2}$ ) showing correlated lesion (arrows) with restricted diffusion, and an ADC value of $0.85 \times 10^{-3} \mathrm{~mm} / \mathrm{s}$. There was no detected abnormal axillary lymphadenopathy on the preoperative MR image; however, metastasis was present in the lymph node specimen after surgery. 

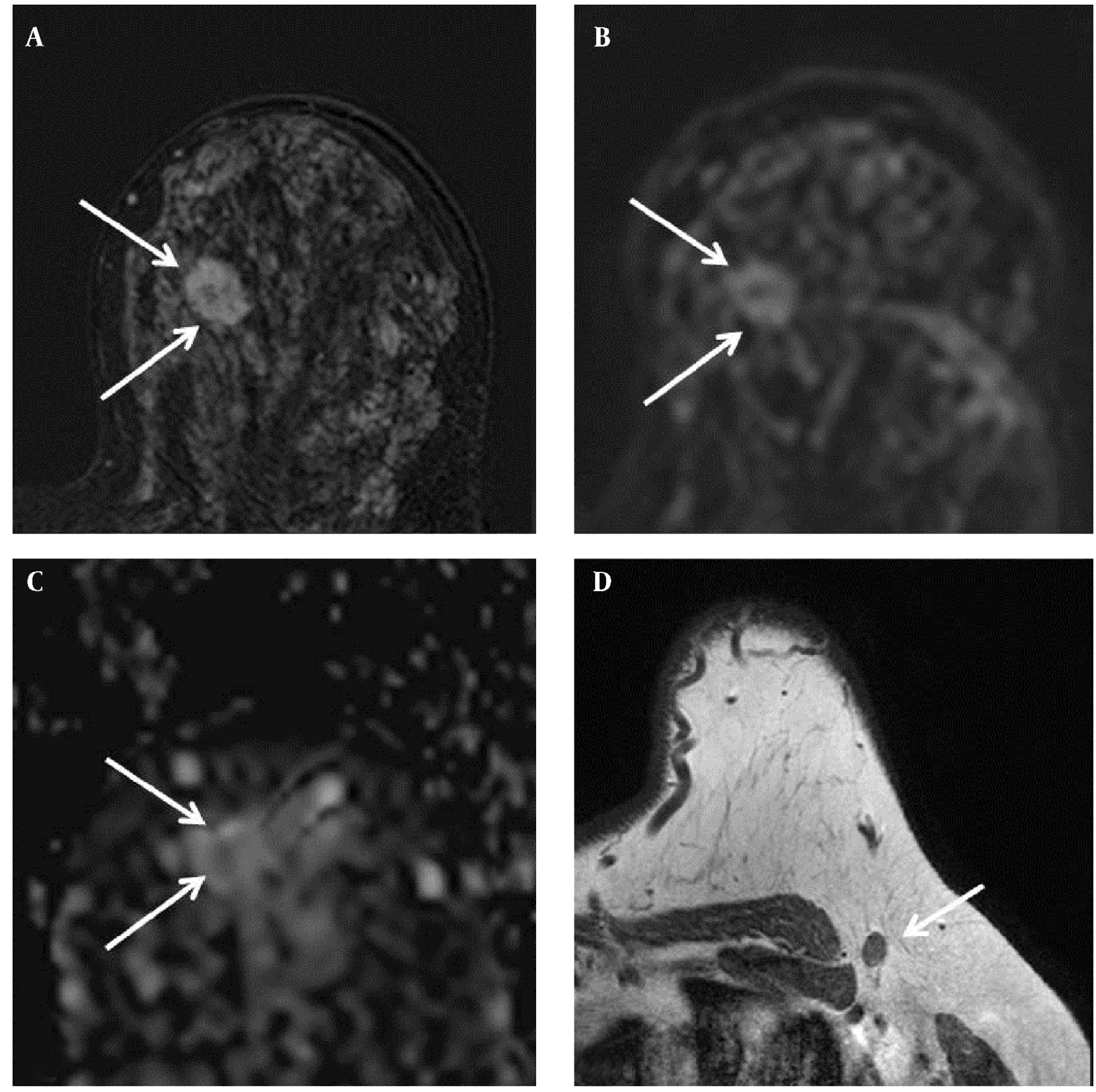

Figure 4. Findings in the right breast of a 44-year-old woman with a diagnosis of left breast cancer. A, Axial contrast-enhanced T1-weighted subtraction MR image of the left breast showing an enhancing mass with an irregular margin (arrows), $19 \times 14 \mathrm{~mm}$ in size; B, Diffusion-weighted image (b value: 1000 $\mathrm{s} / \mathrm{mm}^{2}$ );C, Axial ADC map (b values: 0 and $1000 \mathrm{~s} / \mathrm{mm}^{2}$ ) showing correlated lesion (arrows) with restricted diffusion; the ADC value was $1.08 \times 10^{-3} \mathrm{~mm}^{2} / \mathrm{s}$; D, Axial T1-weighted MR image showing a lymph node with cortical thickening in the left axilla. Metastasis was suspected, but there was no metastasis in the lymph node specimen after excision.

The mean of the p53-positive cancers was higher than that of p53-negative cancers, but there was no significant difference $(\mathrm{P}=0.62)$. Of the molecular subtypes, the HER2 positive type had the highest mean ADC value (mean: $0.99 \pm 0.19 \times$ $10^{-3} \mathrm{~mm}^{2} / \mathrm{s}$ ); however, the ADC values were not significantly different among the molecular subtypes $(P=0.51)$ (Table 1$)$. 
Park EK et al.

\begin{tabular}{|c|c|c|c|c|}
\hline \multirow[t]{2}{*}{ Prognostic Factor Subgroup } & \multirow[t]{2}{*}{ Lesions $^{a}$} & \multicolumn{2}{|c|}{$\operatorname{ADC}\left(\times 10^{-3} \mathrm{~mm}^{2} / \mathrm{s}\right)$} & \multirow[t]{2}{*}{ P Value } \\
\hline & & Mean & 95\% CI & \\
\hline Lymph node status & & & & $<0.01$ \\
\hline Metastasis & $21(30)$ & .83 & $0.77,0.89$ & \\
\hline No metastasis & $50(70)$ & .94 & $0.89,1.00$ & \\
\hline Histological type & & & & 0.19 \\
\hline IDC NOS & $54(76)$ & .89 & $0.84,0.93$ & \\
\hline Non IDC & $17(24)$ & .98 & $0.85,1.12$ & \\
\hline Histological grade & & & & 0.48 \\
\hline Grade 1 & $26(37)$ & .95 & $0.85,1.04$ & \\
\hline Grade 2 & $18(25)$ & .88 & $0.84,0.94$ & \\
\hline Grade 3 & $27(38)$ & .89 & $0.82,0.96$ & \\
\hline Size & & & & 0.46 \\
\hline$<2 \mathrm{~cm}$ & $39(55)$ & .90 & $0.82,0.97$ & \\
\hline$\geq 2 \mathrm{~cm}$ & $32(45)$ & .93 & $0.88,0.98$ & \\
\hline ER & & & & 0.93 \\
\hline Positive & $48(67)$ & .88 & $0.85,0.97$ & \\
\hline Negative & $23(33)$ & .91 & $0.84,0.98$ & \\
\hline PR & & & & 0.88 \\
\hline Positive & $44(62)$ & .91 & $0.84,0.97$ & \\
\hline Negative & $26(38)$ & .92 & $0.85,0.98$ & \\
\hline HER2 & & & & 0.85 \\
\hline Positive & $19(27)$ & .90 & $0.81,0.99$ & \\
\hline Negative & $52(73)$ & .88 & $0.84,0.95$ & \\
\hline Ki-67 & & & & 0.03 \\
\hline Positive & $32(45)$ & .85 & $0.84,0.98$ & \\
\hline Negative & $39(55)$ & .96 & $0.85,0.97$ & \\
\hline p53 & & & & 0.62 \\
\hline Positive & $33(46)$ & .93 & $0.84,0.98$ & \\
\hline Negative & $38(54)$ & .90 & $0.82,0.97$ & \\
\hline Immunohistochemical type & & & & 0.51 \\
\hline Luminal A & $35(49)$ & .93 & $0.86,1.00$ & \\
\hline Luminal B & $13(18)$ & .86 & $0.78,0.95$ & \\
\hline Triple negative & $17(24)$ & .88 & $0.80,0.95$ & \\
\hline HER2 positive & $6(9)$ & .99 & $0.79,1.19$ & \\
\hline
\end{tabular}

Abbreviations: HER2, human epidermal growth factor receptor; IDC, invasive ductal carcinoma; NOS, not otherwise specified.

${ }^{\mathrm{a}}$ Data are presented as No. (\%).

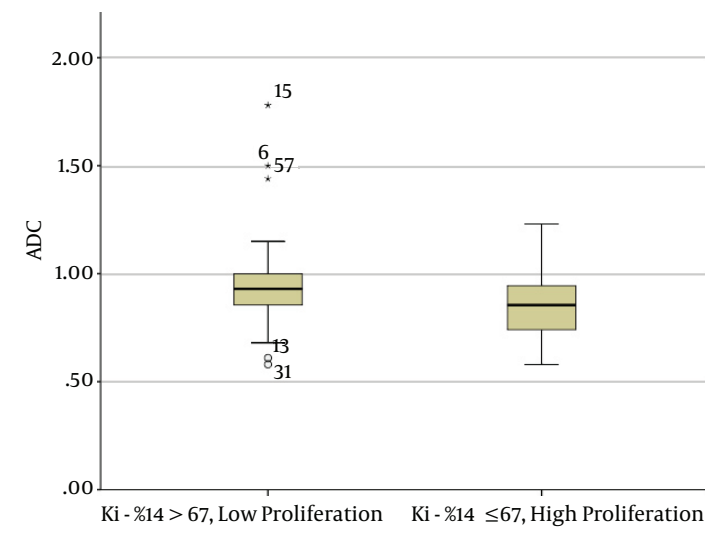

Figure 5. Graph showing the mean ADC values of invasive breast cancers with high proliferation versus low proliferation. The mean ADC value of those with high proliferation (Ki-67: $\geq 14 \% ; 0.85 \pm 0.13 \times 10^{-3} \mathrm{~mm}^{2} / \mathrm{s}$ ) was significantly lower than those with low proliferation (Ki-67: $<14 \%$; $0.96 \pm$ $\left.0.22 \times 10^{-3} \mathrm{~mm}^{2} / \mathrm{s} ; \mathrm{P}=0.03\right)$. The two extreme values in the graph (asterisks) were the ADC values of mucinous carcinomas.

\section{Discussion}

Our prospective study examined consecutively treated patients on whom we performed identical DWIs using a single 3.0 Tesla MR scanner. We found that the ADC is a useful method for the quantitative measurement of the DWI to detect microstructural tumor characteristics. The $\mathrm{ADC}$ values were influenced not only by the microscopic motion from diffusion, but also from perfusion, which could be increased due to tumor angiogenesis $(16,17)$. The perfusion effects were decreased for the higher $b$ values, and diffusion had a more prominent influence on the ADC value $(4,18)$. Many factors can affect the ADC value, including imaging parameters factors, such as magnetic susceptibility, spatial resolution, and the signal-to-noise ratio (6). Therefore, it is important to perform DWIs using a single MR scanner with identical imaging parameters.

Lymph node metastasis is one of the most important prognostic factors in breast cancer patients. Patients 
with lymph node metastasis have about a 4 to 8 times higher mortality rate than those without nodal involvement (18). For the preoperative evaluation of the axilla, several methods, including ultrasound, ultrasound-guided fine-needle aspiration or core biopsy, breast MRI, and positron emission tomography (PET), have been used. In recent years, patients with a negative sentinel lymph node can avoid a complete axillary lymph node dissection, because sentinel lymph node biopsy has been proven to be fairly accurate in predicting axillary metastasis with high sensitivity; however, a false negative result of $5 \%$ has been reported (19).

In this study, the tumors with axillary lymph node metastasis had significantly lower ADCs than those without axillary lymph node metastasis, and the axillary lymph node status was independently associated with the ADC value. These findings are consistent with another prospective study by Razek et al. (10) using a 1.5 Tesla MR scanner. Although some authors have observed no significant relationship between the ADC values and axillary lymph node metastasis $(17,20)$, this approach could contribute to the preoperative detection of axillary lymph node metastasis. Further investigation is necessary to assess the additional roles of DWI in improving the detection rates of axillary lymph node metastases, as well as how to combine imaging modalities for more accurate diagnoses.

A high proliferation rate with a high Ki-67 labeling index was also associated with a low ADC value in this study. The tumor proliferation rate is a key prognostic factor; however, the results obtained in previous studies using different methods of ADC measurement have varied (8, $12,19)$. A recent study by Mori et al. (20) evaluated the association of the Ki-67 labeling index in the luminal type of invasive ductal carcinoma, and found that a high Ki-67 labeling index was associated with the ADC value, regardless of how it was measured.

This study showed no significant association between the ADC values and the ER, PR, and HER2 statuses. One previous study reported that the ER expression affected the ADC value via the inhibition of the angiogenic pathway and a decrease in perfusion (15). Soerjomataram et al. reported that the ER-positive cancers showed high cellularity (21), while Bogner et al. found that HER2 overexpression increased angiogenesis by the induction of the vascular endothelial growth factor (18). Other recent studies, however, have reported inconclusive results on the correlation between the immunohistochemical markers and ADC values. Moreover, Choi et al. using a 1.5 Tesla MRI with b values of 0 and $1000 \mathrm{~s} / \mathrm{mm}^{2}$, reported that a low ADC was associated with a positive expression of ER and PR, but that the expression of HER2 was not significantly associated with the ADC value (8). Jeh et al. used both a 1.5 Tesla MRI with b values of 0 and $1000 \mathrm{~s} / \mathrm{mm} 2$ and 3.0 Tesla MRI with b values of 0 and $750 \mathrm{~s} / \mathrm{mm}^{2}$, and found that the low ADC value was related to the positive expression of ER and negative expression of HER2 (22). A more recent study using a 3.0 Tesla MRI with b values of 0 and $1000 \mathrm{~s} /$ $\mathrm{mm}^{2}$ reported that only the HER2 status was associated with the ADC value (18). However, all of these studies used retrospective study designs with variable b values, which might have influenced the ADC values.

Tumor size is also an important prognostic factor, and is associated with long-term survival from breast cancer. Larger tumors generally have more positive lymph nodes and are associated with a poorer prognosis (21). Razek et al. observed that the mean ADC value was $1.16 \times 10^{-3} \mathrm{~mm}^{2} / \mathrm{s}$ in those tumors measuring 1 to $2 \mathrm{~cm}, 1.01 \times 10^{-3} \mathrm{~mm} 2 / \mathrm{s}$ in those measuring 2 to $5 \mathrm{~cm}$, and $0.95 \times 10^{-3} \mathrm{~mm}^{2} / \mathrm{s}$ in those measuring $>5 \mathrm{~cm}(\mathrm{P}=0.001)(10)$. Park et al. reported that the mean ADC value was $0.91 \times 10^{-3} \mathrm{~mm}^{2} / \mathrm{s}$ in those tumors measuring $<2 \mathrm{~cm}, 0.84 \times 10^{-3} \mathrm{~mm}^{2} / \mathrm{s}$ in those of 2 to $5 \mathrm{~cm}$, and $0.97 \times 10^{-3} \mathrm{~mm}^{2} / \mathrm{s}$ in those $\geq 5 \mathrm{~cm}(\mathrm{P}=0.003)$ (9). These studies $(9,10)$ reported that the tumor size was significantly associated with the ADC value; however, the results for the mean $A D C$ values of the tumors larger than $5 \mathrm{~cm}$ were different.

Our study found that the larger tumors ( $\geq 2 \mathrm{~cm}$ ) had higher mean ADC values $\left(0.93 \pm 0.15 \times 10^{-3} \mathrm{~mm}^{2} / \mathrm{s}\right)$ than the smaller tumors $\left(<2 \mathrm{~cm}, 0.90 \pm 0.23 \times 10^{-3} \mathrm{~mm}^{2} / \mathrm{s}\right)$, without a statistically significant difference, while Choi et al. (8) found no significant association with the tumor size and mean ADC value: the mean ADC value of the larger tumors $\left(>2 \mathrm{~cm}, 0.89 \times 10^{-3} \mathrm{~mm}^{2} / \mathrm{s}\right)$ was lower than that of the smaller tumors $\left(<2 \mathrm{~cm}, 0.92 \times 10^{-3} \mathrm{~mm}^{2} / \mathrm{s}\right)$. The differences in the outcomes may be attributed to the different histopathological types of tumors and different study methods. Most of these studies have concentrated on 1.5 Tesla MRI scanners $(8,10,22)$; however, 3.0 Tesla MRI scanners provide greater lesion delineation in DWI, and small cancers are more clearly visible in DWI at 3.0 Tesla than at 1.5 Tesla (23). Further, our study included patients with invasive cancers of a variety of histopathological types, while Park et al. (9), who did use a 3.0 Tesla scanner, included only invasive ductal carcinomas.

Tumor cellularity is an important index of tumor grade (9); however, the histological grade of breast cancer is assessed by the degree of differentiation by using the Nottingham histological scoring system (3). Some authors have demonstrated an association between the tumor cellularity and ADC values $(4,17)$, but a direct relationship between the tumor cellularity and histological grade has not been proven. Previous studies reported that the histologic grade of breast cancer was not significantly associated with the ADC value $(8,9,22)$, which corresponds with our results, although a recent study by Cipolla et al. (11) found an association between a low ADC value and a high grade of invasive breast cancer.

There were several limitations in this study. First, we excluded patients undergoing neoadjuvant chemotherapy. Therefore, those patients with advanced breast cancer were not included, and selection bias might be present. Second, breast cancer lesions smaller than $5 \mathrm{~mm}$ were excluded. Although the 3.0 Tesla MRI has greater resolution than the 1.5 Tesla, it was difficult to measure the ADC 
value accurately for lesions less than $5 \mathrm{~mm}$ without intercepting adjacent normal tissue or fat.

In conclusion, a low ADC is associated with lymph node metastasis, and a high Ki-67 labeling index in preoperative breast MRIs. The ability of DWI to preoperatively evaluate lymph node status and tumor proliferation in breast cancer, may further improve patient care and help develop a more effective treatment plan. The preoperative assessment of ADC using the 3.0 Tesla DWI may also help reduce false negative diagnoses in lymph node metastasis.

\section{Footnotes}

Authors' Contribution:Study concept and design: Eun Kyung Park, Kyu Ran Cho; acquisition of data: Eun Kyung Park, Ok Hee Woo; analysis and interpretation of data: Eun Kyung Park, Kyu Ran Cho; drafting of the manuscript: Eun Kyung Park, Kyu Ran Cho; critical revision of the manuscript for important intellectual content: Kyu Ran Cho; statistical analysis: Eun Kyung Park, Ok Hee Woo; administrative, technical, and material support: Bo Kyoung Seo, Jeoung Won Bae; study supervision: Kyu Ran Cho, Sung Bum Cho.

Financial Disclosure:The authors report that there are no conflicts of interest.

Funding/Support:This original research did not receive commercial financial support, and the authors have no conflicts of interest.

\section{References}

1. Cheang MC, Chia SK, Voduc D, Gao D, Leung S, Snider J, et al. Ki67 index, HER2 status, and prognosis of patients with luminal B breast cancer. J Natl Cancer Inst. 2009;101(10):736-50. doi:10.1093| jnci/djp082. [PubMed: 19436038]

2. Kennecke H, Yerushalmi R, Woods R, Cheang MC, Voduc D, Speers $\mathrm{CH}$, et al. Metastatic behavior of breast cancer subtypes. J Clin Oncol. 2010;28(20):3271-7. doi: 10.1200/JCO.2009.25.9820. [PubMed: 20498394]

3. Rakha EA, Reis-Filho JS, Baehner F, Dabbs DJ, Decker T, Eusebi V, et al. Breast cancer prognostic classification in the molecular era: the role of histological grade. Breast Cancer Res. 2010;12(4):207. doi:10.1186/bcr2607. [PubMed: 20804570]

4. Guo Y, Cai YQ, Cai ZL, Gao YG, An NY, Ma L, et al. Differentiation of clinically benign and malignant breast lesions using diffusionweighted imaging. J Magn Reson Imaging. 2002;16(2):172-8. doi 10.1002/jmri.10140. [PubMed:12203765]

5. Partridge SC, DeMartini WB, Kurland BF, Eby PR, White SW, Lehman CD. Quantitative diffusion-weighted imaging as an adjunct to conventional breast MRI for improved positive predictive value. AJR Am J Roentgenol. 2009;193(6):1716-22. doi:10.2214/AJR.08.2139. [PubMed: 19933670]

6. Ei Khouli RH, Jacobs MA, Mezban SD, Huang P, Kamel IR, Macura $\mathrm{KJ}$, et al. Diffusion-weighted imaging improves the diagnostic accuracy of conventional 3.0-T breast MR imaging. Radiol ogy. 2010;256(1):64-73. doi: 10.1148/radiol.10091367. [PubMed 20574085]

7. Hosseinzadeh K, Schwarz SD. Endorectal diffusion-weighted imaging in prostate cancer to differentiate malignant and benign peripheral zone tissue. J Magn Reson Imaging. 2004;20(4):654-61. doi:10.1002/jmri.20159. [PubMed: 15390142]

8. Choi SY, Chang YW, Park HJ, Kim HJ, Hong SS, Seo DY. Correla- tion of the apparent diffusion coefficiency values on diffusionweighted imaging with prognostic factors for breast cancer. $\mathrm{Br}$ J Radiol. 2012;85(1016):e474-9. doi: 10.1259/bjr/79381464. [PubMed: 22128125]

9. Park SH, Choi HY, Hahn SY. Correlations between apparent diffusion coefficient values of invasive ductal carcinoma and pathologic factors on diffusion-weighted MRI at 3.0 Tesla. J Magn Reson Imaging. 2015;41(1):175-82. doi: 10.1002/jmri.24519. [PubMed: 24353241]

10. Razek AA, Gaballa G, Denewer A, Nada N. Invasive ductal carcinoma: correlation of apparent diffusion coefficient value with pathological prognostic factors. NMR Biomed. 2010;23(6):619-23. doi:10.1002/nbm.1503. [PubMed:20232453]

11. Cipolla V, Santucci D, Guerrieri D, Drudi FM, Meggiorini ML, de Felice C. Correlation between 3T apparent diffusion coefficient values and grading of invasive breast carcinoma. Eur J Radiol. 2014;83(12):2144-50. doi: 10.1016/j.ejrad.2014.09.015. [PubMed: 25305145]

12. Martincich L, Deantoni V, Bertotto I, Redana S, Kubatzki F, Sarotto I, et al. Correlations between diffusion-weighted imaging and breast cancer biomarkers. Eur Radiol. 2012;22(7):1519-28. doi: 10.1007/s00330-012-2403-8. [PubMed: 22411304]

13. De Felice C, Cipolla V, Guerrieri D, Santucci D, Musella A, Porfir LM, et al. Apparent diffusion coefficient on 3.0 Tesla magnetic resonance imaging and prognostic factors in breast cancer. Eur J Gynaecol Oncol. 2014;35(4):408-14. [PubMed: 25118482]

14. Elston CW, Ellis IO. Pathological prognostic factors in breast cancer. I. The value of histological grade in breast cancer: experience from a large study with long-term follow-up. C. W. Elston \& I. O. Ellis. Histopathology 1991; 19; 403-410. Histopathology. 2002;41(3A):151-2. [PubMed:12405945]

15. Black R, Prescott R, Bers K, Hawkins A, Stewart H, Forrest P. Tumour cellularity, oestrogen receptors and prognosis in breast cancer. Clin Oncol. 1983;9(4):311-8. [PubMed: 6661854]

16. Park MJ, Cha ES, Kang BJ, Ihn YK, Baik JH. The role of diffusionweighted imaging and the apparent diffusion coefficient (ADC) values for breast tumors. Korean J Radiol. 2007;8(5):390-6 [PubMed: 17923781]

17. Woodhams R, Matsunaga K, Iwabuchi K, Kan S, Hata H, Kurana$\mathrm{mi} \mathrm{M}$, et al. Diffusion-weighted imaging of malignant breast tumors: the usefulness of apparent diffusion coefficient (ADC) value and $\mathrm{ADC}$ map for the detection of malignant breast tumors and evaluation of cancer extension.J Comput Assist Tomogr 2005;29(5):644-9. [PubMed:16163035]

18. Bogner W, Gruber S, Pinker K, Grabner G, Stadlbauer A, Weber M, et al. Diffusion-weighted MR for differentiation of breast lesions at 3.0 T: how does selection of diffusion protocols affect diagnosis? Radiology. 2009;253(2):341-51. doi: 10.1148/radiol.2532081718. [PubMed: 19703869]

19. Kim SH, Cha ES, Kim HS, Kang BJ, Choi JJ, Jung JH, et al. Diffusionweighted imaging of breast cancer: correlation of the apparent diffusion coefficient value with prognostic factors. J Magn Reson Imaging. 2009;30(3):615-20. doi: 10.1002/jmri.21884. [PubMed: 19711411]

20. Mori N, Ota H, Mugikura S, Takasawa C, Ishida T, Watanabe G, et al. Luminal-type breast cancer: correlation of apparent diffusion coefficients with the Ki-67 labeling index. Radiology. 2015;274(1):6673. doi: 10.1148/radiol.14140283. [PubMed: 25203132]

21. Soerjomataram I, Louwman MW, Ribot JG, Roukema JA, Coebergh JW. An overview of prognostic factors for long-term survivors of breast cancer. Breast Cancer Res Treat. 2008;107(3):309-30. doi:10.1007/s10549-007-9556-1. [PubMed: 17377838]

22. Jeh SK, Kim SH, Kim HS, Kang BJ, Jeong SH, Yim HW, et al. Correlation of the apparent diffusion coefficient value and dynamic magnetic resonance imaging findings with prognostic factors in invasive ductal carcinoma. JMagn Reson Imaging. 2011;33(1):102-9. doi:10.1002/jmri.22400. [PubMed: 21182127]

23. Matsuoka A, Minato M, Harada M, Kubo H, Bandou Y, Tangoku A et al. Comparison of 3.0-and 1.5-tesla diffusion-weighted imaging in the visibility of breast cancer. Radiat Med. 2008;26(1):15-20. doi:10.1007/s11604-007-0187-6. [PubMed: 18236129] 